\title{
Thermoelectrical detection of Majorana states
}

\author{
Rosa López \\ Institut de Física Interdisciplinària i de Sistemes Complexos IFISC (CSIC-UIB), E-07122 Palma de Mallorca, Spain \\ and Kavli Institute for Theoretical Physics, University of California, Santa Barbara, California 93106-4030, USA \\ Minchul Lee \\ Department of Applied Physics, College of Applied Science, Kyung Hee University, Yongin 446-701, Korea \\ Llorenç Serra \\ Institut de Física Interdisciplinària i de Sistemes Complexos IFISC (CSIC-UIB), E-07122 Palma de Mallorca, Spain \\ and Departament de Física, Universitat de les Illes Balears, E-07122 Palma de Mallorca, Spain \\ Jong Soo Lim \\ School of Physics, Korea Institute for Advanced Study, Seoul 130-722, Korea
}

(Received 29 October 2013; published 19 May 2014)

\begin{abstract}
We discuss the thermoelectrical properties of nanowires hosting Majorana states. For a Majorana nanowire directly coupled to two normal reservoirs the thermopower always vanishes regardless of the value of the Majorana overlap parameter $\varepsilon_{M}$, due to the particle-hole symmetric nature of the Majorana states. This situation changes drastically if a quantum dot (QD) is inserted to break the particle-hole symmetry. Then, the Majorana-side-coupled QD system exhibits a different behavior for the thermopower depending on the value of $\varepsilon_{M}$. The thermopower globally reverses its sign when the half-fermionic nature of the Majorana state is relevant, i.e., for $\varepsilon_{M} \lesssim k_{B} T_{b}$, where $T_{b}$ is the background temperature. For large overlap, $\varepsilon_{M} \gtrsim k_{B} T_{b}$, on the other hand, the Seebeck coefficient behaves similarly to that in a resonant level system. The sign change of the thermopower and the fact that both the electrical and thermal conductances reach their half-fermionic value when Majorana physics are maximal could serve as a proof of the existence of Majorana states in nanowires.
\end{abstract}

DOI: 10.1103/PhysRevB.89.205418

PACS number(s): 72.15.Jf, 73.21.La, 78.67.Uh, 72.10.Fk

\section{INTRODUCTION}

Nowadays there is much interest in the interplay between heat and charge flows in nanostructures [1,2]. Thermovoltages generated in response to a temperature gradient have been shown to be much bigger at the nanoscale due to the peculiar properties of quantum systems [3-7]. For example, a delta-like density of states occurring in confined nanostructures such as quantum wells [3] can alter dramatically their thermolectrical properties. The main utility of thermoelectrical devices is the heat-to-electricity conversion processes. From a more fundamental point of view, however, both thermal and electrical transports reveal information on the intrinsic nature of a quantum system. One example is the departure from the Wiedemann-Franz law attributed to non-Fermi-liquid behavior [8]. In addition, thermoelectric transport measurements are able to distinguish between distinct types of carriers, such as electrons and holes in Andreev systems [9,10] and molecular junctions [11].

Our motivation is to address to what extent Majorana physics can be reflected in the thermoelectrical transport properties of a system. The unambiguous detection of Majorana fermions in solid state devices is still an issue under discussion. Majorana physics, in the low-energy domain, was predicted to occur as quasiparticle excitations [12]. The first proposals suggested their observation in quantum Hall states, e.g., the Moore-Read state at filling factor $v=5 / 2$ [13]. Then, other suggestions considered some exotic superconductors such as $\mathrm{Sr}_{2} \mathrm{RuO}_{4}$ or $p$-wave superconductors [14-17]. Later on, the pioneering work by $\mathrm{Fu}$ and Kane [18] demonstrated that such quasiparticles could be created in a topological insulator brought in close proximity to a superconductivity source. However, the Majorana search has been very prolific in the realm of quasi-one-dimensional semiconductor nanowires [19-23], particularly in large $g$-factor materials such as InAs and InSb. Most of the experiments designed to detect these elusive quasipartices have been performed via electrical transport measurements [24-28] by tunnel spectroscopy. A voltage shift $\delta V$ is applied to the nanowire ends, which generates an electrical current $I$. The Majorana signature appears as a zero-bias anomaly in the nonlinear conductance $d I / d V$ [29-32]. In semiconductor nanowires, Majorana quasiparticles arise when superconductivity (source of electrons and holes), strong spin-orbit interaction, and magnetic field work together. Then, under certain conditions the nanowire enters into the named topological phase and reveals spinless and chargeless zero-energy states, i.e., very elusive quasiparticle excitations. We refer to this as Majorana nanowire. However, the presence of a zero-bias anomaly in the nonlinear conductance does not warrant the presence of Majorana quasiparticles. Kondo physics can be observed in normal superconductor nanowires as well [33,34]. Furthermore, nearly zero-energy Andreev states [35,36] or weak antilocalization [37] effects are possible sources of zero-bias anomaly in normal superconductor nanowires. There are other suggestions to detect Majorana zero-energy states in Josephson junctions and rings [38-46]. The Josephson current displays an anomalous periodicity of $4 \pi$ if Majorana physics takes place. However, so far the experimental verification is not yet definitive [47]. 
(a)

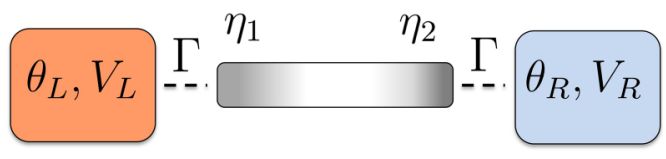

(b)

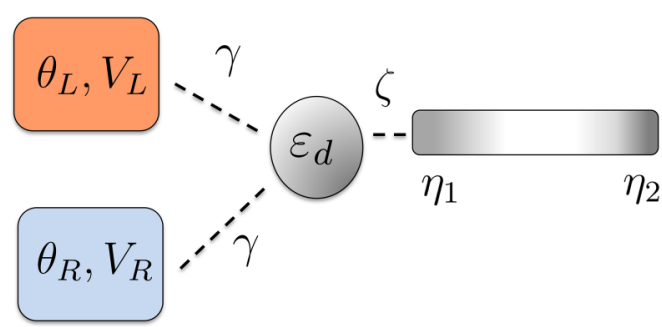

FIG. 1. (Color online) (a) Majorana nanowire tunnel-coupled to two normal contacts by tunneling barriers of probability $\Gamma$. Here, $\eta_{1}$, and $\eta_{2}$ denote the two Majorana states formed at the ends of the nanowire. The left (right) metallic contact is electrically and thermally biased by $V_{L(R)}$ and $\theta_{L(R)}$. (b) A quantum dot is inserted and symmetrically coupled to the metallic reservoirs with tunneling rate $\gamma$. One end of the Majorana nanowire is side-coupled to the dot, and such coupling is characterized by the parameter $\zeta$.

Our goal consists in utilizing the thermoelectrical properties as a tool to detect the presence of Majorana states formed in the ends of topological superconductor nanowires. There are earlier proposals of using thermopower measurements to probe the non-Abelian anyons in the Moore-Read state $[48,49]$ which have been recently tested in the fractional quantized Hall regime [50]. Then, quite recently, detection of Majorana zero modes in $p$-wave superconductors was proposed [51]. Here, we propose a way of detecting Majorana states in semiconductor nanowires by applying a temperature gradient $\left(\delta \theta=\theta_{L}-\theta_{R}\right)$ which in turn generates an induced electrical shift $\left(\delta V=V_{L}-V_{R}\right)$. We analyze two-terminal devices as depicted in Fig. 1 and determine both the electrical and energy currents. In Fig. 1, the Majorana nanowire is contacted to two normal reservoirs. In general, the linear-response electric $I$ and energy $J$ currents can be expressed as

$$
\left(\begin{array}{l}
I \\
J
\end{array}\right)=\left(\begin{array}{ll}
G & L \\
M & K
\end{array}\right)\left(\begin{array}{l}
\delta V \\
\delta \theta
\end{array}\right) .
$$

The $2 \times 2$ matrix is the Onsager matrix that includes diagonal elements, the electric $G$ and thermal $K$ conductances, and nondiagonal coefficients, the thermoelectric $L$ and electrothermal $M$ conductances. The two latter are related due to microreversibility condition [52,53]. More specifically, we are interested in the determination of the Seebeck coefficient or thermopower that quantifies the conversion of heat into electricity in thermoelectrical machines. The Seebeck coefficient is easily determined from the relation $S=-\delta V /\left.\delta \theta\right|_{I=0}=L / G$ (derived from the open circuit condition for the charge flow).

Our results for a two-terminal Majorana nanowire [see Fig. 1(a)] show that both $G$ and $K$ reach their maximum value only when two Majorana states do not overlap. On the contrary, the thermoelectrical (electrothermal) response always vanishes irrespectively of the Majorana overlap strength. As a result, the Seebeck coefficient vanishes owing to the intrinsic particlehole symmetry of the Majorana states. However, this physical scenario can be dramatically altered by inserting a quantum dot in between the two normal contacts and side-coupled to the Majorana nanowire [see Fig. 1(b)] [54-56]. In this arrangement, the Seebeck coefficient can be tuned by gating the dot, i.e., $S=S\left(\varepsilon_{d}\right)$ with $\varepsilon_{d}$ the dot level position. We find that the thermopower globally reverses its sign for $\varepsilon_{M} \lesssim k_{B} T_{b}$, where $T_{b}$ is the background temperature. For large overlap, $\varepsilon_{M} \gtrsim k_{B} T_{b}$, on the other hand, $S$ behaves similarly to that in a resonant level system as we will discuss later. The sign change of the thermopower and the fact that both the electrical and thermal conductances reach their half-fermionic value when Majorana physics are maximal could serve as a proof of the existence of Majorana states in nanowires.

\section{TWO-TERMINAL MAJORANA JUNCTION}

We present our theory for the thermoelectrical transport by employing the nonequilibrium Keldysh Green function framework. We consider a semiconductor nanowire with strong Rashba spin-orbit interaction with proximity induced $s$-wave superconductivity, and an applied magnetic field $B$. We assume a sufficiently long wire to neglect charging effects. The magnetic field is such that the wire is in the topological phase, $\Delta_{Z}>\sqrt{\Delta^{2}+\mu^{2}}$, with $\Delta_{Z}=g \mu_{B} B / 2, \Delta$ the superconducting gap, and $\mu$ the wire chemical potential. The topological phase in the nanowire can be described by a low-energy effective model where the two Majorana zero-energy states are represented by the Majorana operators $\eta_{1}=f+f^{\dagger}$, and $\eta_{2}=i\left(f^{\dagger}-f\right)$ (in terms of a Dirac fermion operator $f$ ). $\eta_{\beta}$ follows the Clifford algebra $\left\{\eta_{\beta}, \eta_{\beta^{\prime}}\right\}=2 \delta_{\beta \beta^{\prime}}$, where $\eta_{\beta}=\eta_{\beta}^{\dagger}$. Two normal contacts are tunnel-coupled to the two ends of the wire, respectively, as shown in Fig. 1(a). The total Hamiltonian describing our system consists of three contributions: $\mathcal{H}=\mathcal{H}_{C}+\mathcal{H}_{M}+\mathcal{H}_{T}$, where

$$
\begin{aligned}
& \mathcal{H}_{C}=\sum_{\alpha, k} \varepsilon_{\alpha k} c_{\alpha k}^{\dagger} c_{\alpha k}, \quad \mathcal{H}_{M}=\frac{i}{2} \varepsilon_{M} \eta_{1} \eta_{2}, \\
& \mathcal{H}_{T}=\mathcal{H}_{T L}+\mathcal{H}_{T R}=\sum_{\alpha, k, \beta}\left[V_{\alpha k, \beta}^{*} c_{\alpha k}^{\dagger} \eta_{\beta}+(\text { H.c. })\right] .
\end{aligned}
$$

Here, $\mathcal{H}_{C}$ describes the two normal leads with $c_{\alpha k}$ being the conduction-electron operator with wavevector $k$ in lead $\alpha=L, R . \mathcal{H}_{M}$ characterizes the coupling between the two Majorana states where the overlap amplitude $\varepsilon_{M}$ depends on the wire parameters: $\varepsilon_{M} \sim f(B, \Delta) e^{-L / \xi_{0}}$ with $L$ the length of the wire, $\xi_{0}$ the superconducting coherence length, and $f(B, \Delta)$ a complicated function of $B$ and $\Delta$ that determines $\varepsilon_{M}$. For our purpose we assume that $\varepsilon_{M}$ is a controllable parameter. The last contribution, $\mathcal{H}_{T}$, corresponds to the tunnel Hamiltonian between normal leads and the Majorana states. As a good approximation, we assume that the lead electron only hybridizes with the nearest Majorana end. It is because the lead-Majorana tunnel coupling decreases exponentially with the distance and we can safely neglect the tunnel amplitude between the lead and the farthermost Majorana state. Then, assuming that the tunneling is energy independent, the tunnel amplitude $V_{\alpha k, \beta}$ is taken as $V_{0}$ for $\alpha=\beta$ and zero for $\alpha \neq \beta$. This defines the hybridization amplitude $\Gamma=\pi V_{0}^{2} \rho_{0}$, with $\rho_{0}$ the contact density of states. 
It is quite straightforward to express the charge and energy currents through the wire in the Landauer-Büttiker forms, respectively,

$$
I=\frac{e}{h} \int d \omega \mathcal{T}(\omega)\left[f_{L}(\omega)-f_{R}(\omega)\right],
$$

and

$$
J=\frac{1}{h} \int d \omega \omega \mathcal{T}(\omega)\left[f_{L}(\omega)-f_{R}(\omega)\right],
$$

with a transmission coefficient given by

$$
\mathcal{T}(\omega)=\frac{4 \Gamma^{2}\left(\omega^{2}+4 \Gamma^{2}+\varepsilon_{M}^{2}\right)}{\left(\omega^{2}+4 \Gamma^{2}\right)^{2}+\varepsilon_{M}^{2}\left[\varepsilon_{M}^{2}-2\left(\omega^{2}-4 \Gamma^{2}\right)\right]},
$$

and $e(>0)$ the electron charge. Here $f_{\alpha}(\omega)=1 /[1+$ $\left.\exp \left(\omega-\left(\mu-e V_{\alpha}\right)\right) / k_{B} \theta_{\alpha}\right]\left(k_{B}\right.$ : Boltzmann constant) is the Fermi-Dirac distribution function for lead $\alpha$ with $V_{L, R}=$ $\pm \delta V / 2$ and $\theta_{L, R}=T_{b} \pm \delta \theta / 2$ with $T_{b}$ as the background temperature.

In the linear-response limit, the coefficients in Eq. (1) are obtained by (taking $\mu=0$ )

$$
\begin{aligned}
G & =\frac{e^{2}}{h} \int d \omega \mathcal{T}(\omega)\left[-\frac{\partial f_{e q}}{\partial \omega}\right], \\
L & =\frac{e}{h T_{b}} \int d \omega \omega \mathcal{T}(\omega)\left[-\frac{\partial f_{e q}}{\partial \varepsilon}\right], \\
M & =\frac{e}{h} \int d \omega \omega \mathcal{T}(\omega)\left[-\frac{\partial f_{e q}}{\partial \omega}\right], \\
K & =\frac{1}{h T_{b}} \int d \omega \omega^{2} \mathcal{T}(\omega)\left[-\frac{\partial f_{e q}}{\partial \omega}\right],
\end{aligned}
$$

where $f_{e q}$ is the Fermi-Dirac distribution function in equilibrium $(\delta \theta=0$ and $\delta V=0)$. In a Sommerfeld expansion, at sufficiently low temperatures, the linear response conductances $G$ and $K$ have the same behavior with the transmission coefficient up to proportionality factors $G_{0}$ and $K_{0}$. Thus,

$$
G=\lim _{\delta V \rightarrow 0} \frac{d I}{d V}=G_{0} \frac{4 \Gamma^{2}}{\varepsilon_{M}^{2}+4 \Gamma^{2}}
$$

and

$$
K=\lim _{\delta \theta \rightarrow 0} \frac{d J}{d \theta}=K_{0} \frac{4 \Gamma^{2}}{\varepsilon_{M}^{2}+4 \Gamma^{2}},
$$

with $G_{0}=e^{2} / h$ (quantum electrical conductance) and $K_{0}=$ $\pi^{2} k_{B}^{2} T_{b} / 3 h$ (quantum thermal conductance). They take their maximum values $G_{0}$ and $K_{0}$, respectively, when $\varepsilon_{M}=0$ and they decrease as $\varepsilon_{M}$ grows. Importantly, the off-diagonal conductances are always zero, $L=L_{0} \partial \mathcal{T}(\omega) /\left.\partial \omega\right|_{\omega=0}$ with $L_{0}=e \pi^{2} k_{B}^{2} T_{b} / 3 h$ (and $M=L / T_{b}$ ). The vanishing of $L(M)$ has profound consequences in the thermopower or Seebeck coefficient (we recall that $S=L / G$ ). The Seebeck coefficient vanishes regardless of the value of $\varepsilon_{M}$. The reason for this result lies in the inherent particle-hole symmetry of the Majorana states. Hence there is no electric response to a thermal gradient.

\section{SIDE TUNNEL-COUPLED DOT-MAJORANA SYSTEM}

Asymmetry in the particle and hole subspaces can be brought into the system by inserting a quantum dot between the two normal contacts and connecting the dot to one of the Majorana states, as illustrated in Fig. 1(b). The thermoelectrical transport through the dot-Majorana system can then exhibit a nonzero value for the off-diagonal Onsager conductances when the dot is off resonance, giving rise to a nonzero Seebeck coefficient.

In order to include the quantum dot we need to reformulate the Hamiltonian as follows. Under strong magnetic field which is applied to induce the topological phase in the wire, the quantum dot can be regarded as a spin-polarized (or spinless) dot with a single dot level $\varepsilon_{d}$, being described by the dot Hamiltonian

$$
\mathcal{H}_{d}=\varepsilon_{d} d^{\dagger} d
$$

where the $d$ operator annihilates an electron with energy $\varepsilon_{d}$ on the dot. Our system is then mapped onto a resonant level model where the resonant state is coupled to two normal-metal contacts and a Majorana state at one end of the nanowire. The dot-lead tunneling is described by

$$
\mathcal{H}_{T d}=\sum_{\alpha k}\left(W_{\alpha k} c_{\alpha k}^{\dagger} d+\text { H.c. }\right) .
$$

For simplicity, we assume a symmetric and energyindependent dot-lead coupling with a common tunneling rate, $\gamma=\pi W_{0}^{2} \rho_{0}$, with $W_{\alpha k}=W_{0}$. The dot-Majorana sidecoupling Hamiltonian reads

$$
\mathcal{H}_{T M}=\sum_{\beta=1,2} \zeta_{\beta}\left(d^{\dagger} \eta_{\beta}+\text { H.c. }\right)
$$

Here, we assume that only the closest Majorana state to the dot is strongly tunnel coupled, say $\eta_{1}: \zeta_{1}=\zeta$ and $\zeta_{2}=0$. The total Hamiltonian is the sum of all these contributions: $\mathcal{H}=\mathcal{H}_{C}+\mathcal{H}_{d}+\mathcal{H}_{M}+\mathcal{H}_{T d}+\mathcal{H}_{T M}$ [refer to Eq. (2) for the contact and Majorana Hamiltonians, $\mathcal{H}_{C}$ and $\mathcal{H}_{M}$ ]. Now, the charge and energy flows can be expressed in terms of the dot transmission (see Ref. [55] for details)

$$
\mathcal{T}_{d}(\omega)=-2 \gamma \operatorname{Im} \mathcal{G}_{d}^{r}(\omega),
$$

where $\mathcal{G}_{d}^{r}$ is the retarded dot Green function

$$
\mathcal{G}_{d}^{r}(\omega)=\frac{1}{\omega-\varepsilon_{d}+2 i \gamma-B(\omega)[1+\tilde{B}(\omega)]},
$$

with

$$
\tilde{B}(\omega)=\frac{B(\omega)}{\omega+\varepsilon_{d}+2 i \gamma-B(\omega)} .
$$

The parameter $\zeta$ in Eq. (12) characterizes the dot Majorana coupling where $B(\omega)=|\zeta|^{2} /\left(\omega-\varepsilon_{M}^{2} / \omega\right)$ is the dot Majorana self-energy.

\section{DISCUSSION}

Before starting the discussion of the thermoelectrical properties in the dot Majorana system, it is worth revisiting the behavior of the dot transmission with respect to the system parameters, $\varepsilon_{M}, \varepsilon_{d}, \zeta$, and $\gamma$ [55]. Hereafter, we employ $D=$ 50 for the contact bandwidth that determines our energy unit.

Figure 2 illustrates the dependence of $\mathcal{T}_{d}(\omega)$ on $\zeta$ and $\gamma$ when the dot is on resonance and no Majorana overlap occurs $\left(\varepsilon_{d}=\varepsilon_{M}=0\right)$. At $\zeta=0$, the transmission corresponds to the 


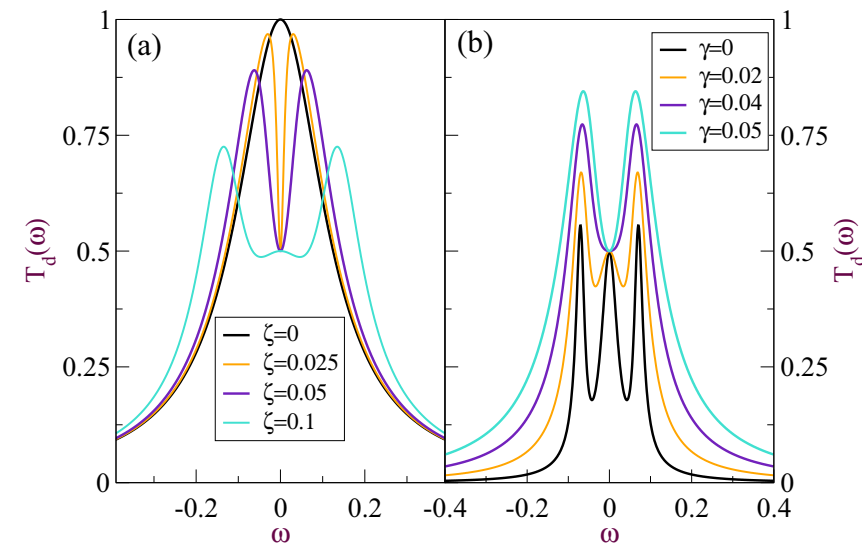

FIG. 2. (Color online) Dot transmission $\mathcal{T}_{d}(\omega)$ (a) for $\gamma=0.06$ and various $\zeta$ values as indicated and (b) for different $\gamma$ values and $\zeta=0.05$. Here the values of the other parameters are set to $\varepsilon_{d}=0$ and $\varepsilon_{M}=0$.

resonant level model with unitary transmission. As $\zeta$ is turned on, two peaks at $\omega= \pm \zeta$ appear due to the dot-Majorana hybridization [see Fig. 2(a)]. Now, keeping $\zeta$ fixed and tuning $\gamma$, the dot transmission evolves to a three-peak structure when $\gamma \approx \zeta$, in which the zero-energy peak is the signature of the presence of Majorana edge states [see Fig. 2(b)]. In all cases, as long as $\zeta \neq 0$, the dot transmission at $\omega=0$ is always half fermionic $[55,56]$. As the Majorana overlap becomes finite $\left(\varepsilon_{M} \neq 0\right), \mathcal{T}_{d}(\omega=0)$ becomes unitary, as shown in Fig. 3(a). For large $\varepsilon_{M}, \mathcal{T}_{d}$ resembles with that for a resonant level model, with resonances at $\omega \approx \pm \varepsilon_{M}$ due to the coupling of the dot state with the $f$ Dirac fermions in the wire (resulting from the large Majorana overlap).

Thermoelectrical effects appear when the transmission becomes asymmetric as can be inferred in the conductance expression, Eq. (6b). In order to observe such asymmetric transmission between positive and negative frequencies the dot level must be positioned off resonance, i.e., $\varepsilon_{d} \neq 0$. This situation is presented in Fig. 3(b) for several values of $\varepsilon_{M}$ for $\varepsilon_{d}>0$. The transmission now features quite an asymmetric structure even for $\varepsilon_{M}=0$. An important feature in $\mathcal{T}_{d}(\omega)$ is

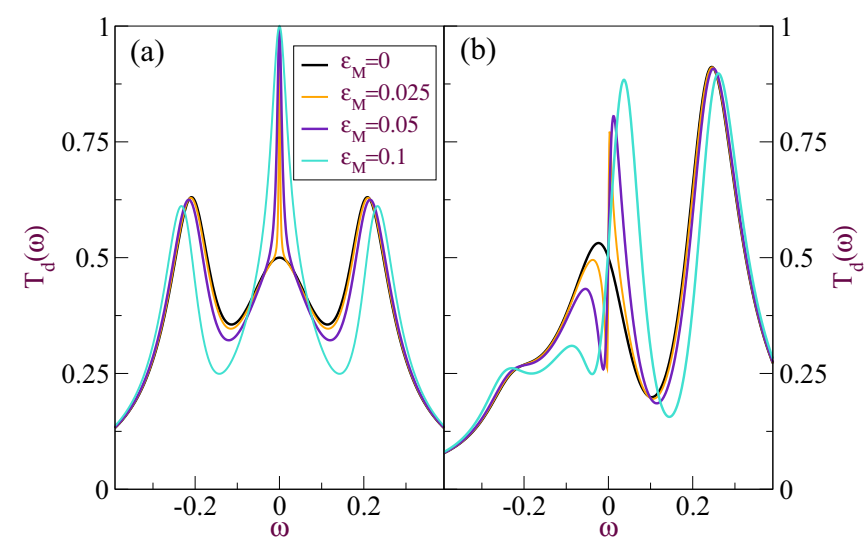

FIG. 3. (Color online) Dot transmission $\mathcal{T}_{d}(\omega)$ for different values of the Majorana overlap $\varepsilon_{M}$ (a) for $\varepsilon_{d}=0$ and (b) for $\varepsilon_{d}=0.12$. Here we have used $\gamma=0.06$ and $\zeta=0.15$.

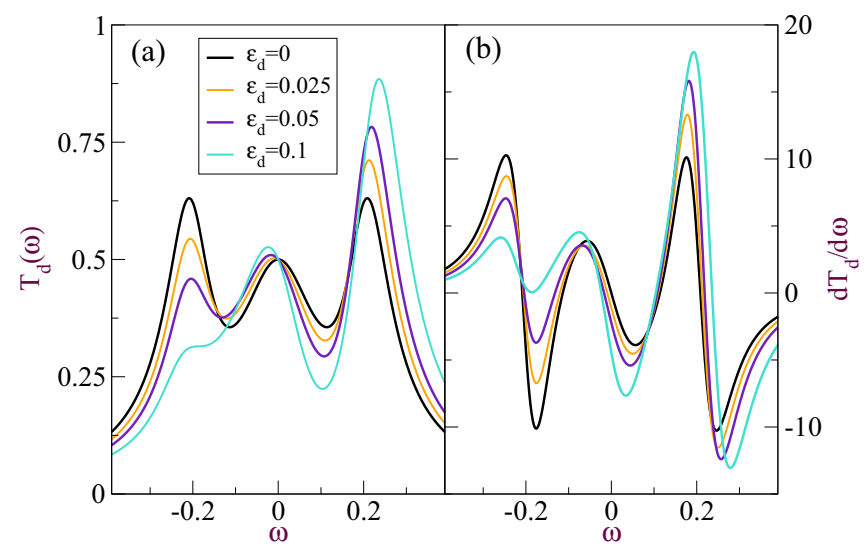

FIG. 4. (Color online) (a) Dot transmission $\mathcal{T}_{d}(\omega)$ and (b) its derivative $\partial \mathcal{T}_{d}(\omega) / \partial \omega$ for the indicated values of $\varepsilon_{d}$ and $\varepsilon_{M}=0$. Here we have set $\gamma=0.06$ and $\zeta=0.15$.

the shift of the central peak near $\omega=0$ with increasing $\varepsilon_{M}$ : At $\varepsilon_{M}=0$, the peak is at the negative $\omega$ side but it moves toward the positive side as $\varepsilon_{M}$ increases. This shift can be understood in terms of the hybridization of the Majorana state (at $\varepsilon_{M}$ ) and the dot level (at $\varepsilon_{d}$ ). In the context of the perturbation theory, the coupling between them repels each other: Since $\varepsilon_{M}<\varepsilon_{d}$ the Majorana level goes lower than the unperturbed level. It explains the appearance of the peak in the negative side for $\varepsilon_{M}=0$. As $\varepsilon_{M}$ increases, the perturbed level also slowly follows the unperturbed level, eventually going to the positive side. It should be noted that the shift will be reversed for $\varepsilon_{d}<0$ in a similar way. This shift of the central peak determines the sign of the thermoelectric conductance $L$ at low temperatures, which will be discussed later.

The dot gate dependence of $\mathcal{T}_{d}(\omega)$ for an ideal Majorana nanowire $\left(\varepsilon_{M}=0\right)$ is depicted in Fig. 4(a) and its energy derivative in Fig. 4(b). These curves show that the transmission at zero energy is always half fermionic as should be for $\varepsilon_{M}=0$, regardless of the dot gate value. However, it is interesting to observe that the energy derivative of the transmission at zero energy acquires some dot gate dependence reflecting the asymmetry between the particle and hole sectors. This result is important for the thermoelectrical conductance $L$ : we recall that at low temperatures $L=L_{0} \partial T_{b}(\omega) /\left.\partial \omega\right|_{\omega=0}$, implying that $L$ becomes gate dependent. Whereas the diagonal conductances are not sensitive to the particle-hole asymmetry introduced by nonzero $\varepsilon_{d}$, the off-diagonal conductances show a dot gate dependence, affecting significantly the thermoelectrical transport.

Our previous analysis for the dot transmission explains the curves for the conductances illustrated in Fig. 5. Both the electric and thermal conductances, $G$ and $K$, depend strongly on $\varepsilon_{d}$ for finite $\varepsilon_{M} \gtrsim k_{B} T_{b}$. Otherwise, in the ideal situation where $\varepsilon_{M}=0, G$ and $K$ are half fermionic and immune to $\varepsilon_{d}$ [55-57]. This important result serves us to detect the Majorana states in side-coupled dot nanowires systems. However, the previous results are applicable only for purely electrical or thermal transport measurements.

Since we are interested more in the thermolectrical signatures of the Majorana states, we analyze how the off-diagonal conductances behave with the dot gate values. We find that for 


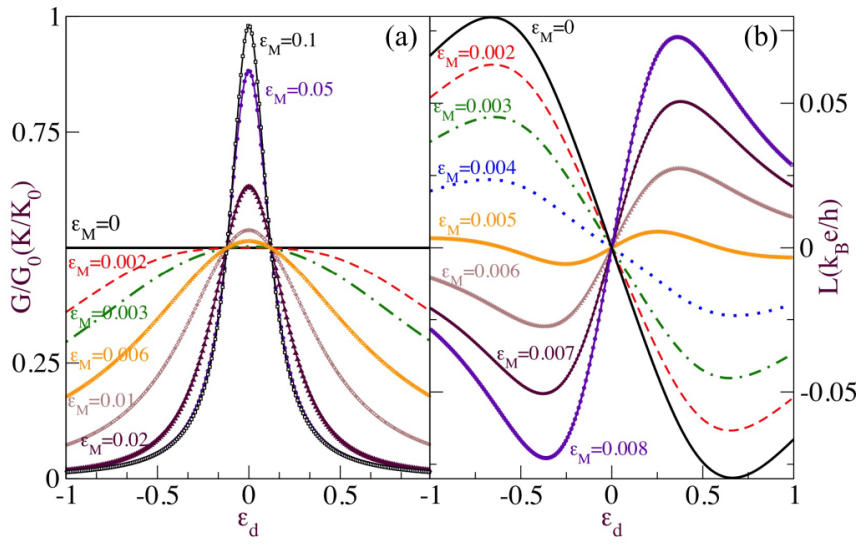

FIG. 5. (Color online) (a) Dot gate dependence of the linear electric conductance $G$ and thermal conductance $K$ for the annotated values of $\varepsilon_{M}$. Here, $G$ is given in units of the quantum of the electrical conductance, $G_{0}=e^{2} / h$, whereas the thermal conductance is given in terms of the quantum of the thermal conductance, $K_{0}=\pi^{2} k_{B}^{2} T_{b} / 3 h$. (b) Thermoelectric conductance $L$ versus $\varepsilon_{d}$ at different values of $\varepsilon_{M}$. Here we have used $\gamma=0.06, \zeta=0.15$, and $k_{B} T_{b}=0.0025$.

small Majorana overlap in comparison with the background temperature, i.e., for $\varepsilon_{M} \lesssim k_{B} T_{b}$, the overall off-diagonal conductance $L(M)$ changes its sign globally (for all values of $\varepsilon_{d}$ ) in comparison with a situation of large Majorana overlap. Such transition is clearly illustrated in Fig. 5. The sign change of $L$ can be also understood in terms of the shape of the transmission. Before, we found that for $\varepsilon_{d}>0$ the central peak in $\mathcal{T}_{d}$ is in the negative $\omega$ side at $\varepsilon_{M}=0$ and shifts toward the positive $\omega$ side with increasing $\varepsilon_{M}$. It explains the sign change of $L$ from negative to positive for $\varepsilon_{d}>0$. For $\varepsilon_{d}<0$, the shift of the peak, starting in the positive side, is reversed so that the sign of $L$ changes from positive to negative.

To gain more insight, an analytical expression for $L$ can be obtained within the Sommerfeld approximation (valid at rather low temperatures). Then, $L$ increases linearly with $\varepsilon_{d}$ and depends inversely on the dot Majorana strength with a negative slope $-1 / 2 \zeta^{2}$ for zero Majorana overlap. Otherwise, for a finite Majorana overlap $\left(\varepsilon_{M} \neq 0\right), L / L_{0}=\left[\varepsilon_{d} /\left(4 \varepsilon_{d}^{2}+\right.\right.$ $\left.\left.\gamma^{2}\right)^{2}\right]\left[8 \gamma^{2}\left(\varepsilon_{M}^{2}+\zeta^{2}\right) / \varepsilon_{M}^{2}\right]$ displays two extrema at $\varepsilon_{d}= \pm \gamma / 2$. In this case, $L$ behaves similarly to that for resonant level model. Note that indeed the sharp transition observed in the Sommerfeld approximation becomes a crossover when $\varepsilon_{M}$ varies on a scale of $k_{B} T_{b}$, as shown in Fig. 5. As long as the temperature is lowered the crossover becomes more and more abrupt.

The sign-change behavior found for the gate dependence of the thermoelectric conductance $L$ is consistent with the presence of Majorana physics. More importantly, the fact that $L$ is an odd function of $\varepsilon_{d}$ for any value of $\varepsilon_{M}$ is a strong evidence of the Majorana state since it reflects the particle-hole symmetric nature of the Majorana states. If one replaces the side-coupled Majorana state by an ordinary fermionic state, then $L$ is no longer an odd function and no global change of sign is observed as $\varepsilon_{M}$ increases.

Using the previous results, we discuss the gate dependence of the thermopower $S=L / G$. Within the range of validity

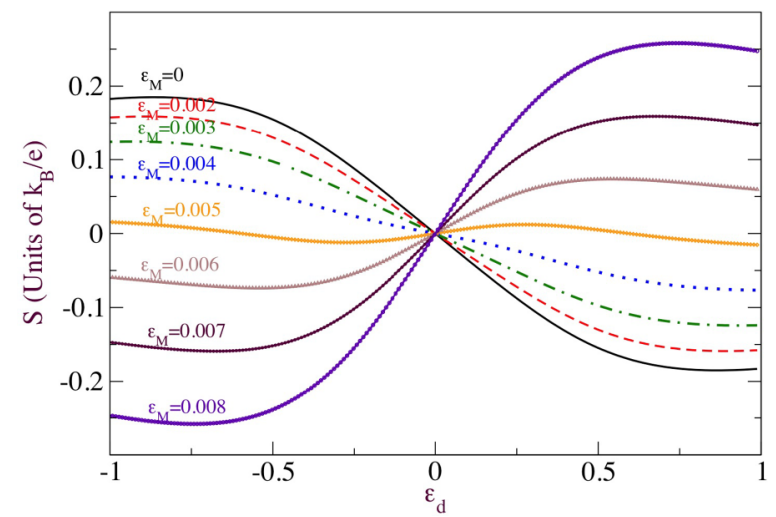

FIG. 6. (Color online) Thermopower $S$ versus $\varepsilon_{d}$ for various values of $\varepsilon_{M}$. Here we have used $\gamma=0.06, \zeta=0.15$, and $k_{B} T_{b}=$ 0.0025 .

of the Sommerfeld expansion, at very low temperatures, we can obtain analytical results for the Seebeck coefficient: $S=$ $S_{0} d \ln \mathcal{T}(\omega) /\left.d \omega\right|_{\omega=0}$ is the Mott formula where we have defined $S_{0}=\pi^{2} k_{B}^{2} T_{b} / 3 e$. For the dot Majorana uncoupled case $(\zeta=0)$, the thermopower $S / S_{0}=8 \varepsilon_{d} /\left(4 \varepsilon_{d}+\gamma^{2}\right)$ vanishes when $\varepsilon_{d}=0$ and follows the resonant level model as expected. For finite $\zeta \neq 0$, remarkably, the thermopower is linear with $\varepsilon_{d}$ for $\varepsilon_{M}=0: S / S_{0}=-\varepsilon_{d} / \zeta^{2}$. The dot gate dependence of $S$ is due to the particle-hole asymmetry introduced when $\varepsilon_{d}$ is tuned from the on to the off resonance situation. This result can be understood by considering two effects. First, the Majorana state contributes to the thermopower in a rigid way with a constant term $-1 / \zeta^{2}$. Second, the particle-hole asymmetry grows with increasing $\left|\varepsilon_{d}\right|$, and it explains why the thermopower is proportional to $\varepsilon_{d}$. Then, both features add up and produce a linear dependence of the Seebeck coefficient on the dot gate with a negative slope that depends on the inverse of the dot Majorana coupling $\zeta$.

Figure 6 plots the Seebeck coefficient $S$ versus $\varepsilon_{d}$ when $k_{B} T_{b}=0.0025$ by numerically integrating Eqs. (6a) and (6b) without assuming the Sommerfeld approximation. We observe that for $\varepsilon_{M} \lesssim k_{B} T_{b}$ the thermopower is positive for negative $\varepsilon_{d}$ having $\delta V<0$ by heating up the left contact. On the contrary, when $\varepsilon_{d}>0$ the thermopower is negative $(S<0)$ leading to a negative potential difference by cooling down the left contact.

The thermopower sign dependence with $\varepsilon_{d}$ is inverted when the Majorana overlap is sufficiently high. Specifically, for $\varepsilon_{M} \gtrsim k_{b} T_{b}$, the thermopower corresponds to the one for a resonant level model $\left.S / S_{0}=\left[\varepsilon_{d} /\left(4 \gamma^{2}+\varepsilon_{d}^{2}\right)\right]\left[8\left(\varepsilon_{M}^{2}+\zeta^{2}\right) / \varepsilon_{M}^{2}\right)\right]$. This means that when $\varepsilon_{d}<0$ the heating of the left contact induces a positive voltage difference whereas for $\varepsilon_{d}<0$ the same voltage difference is generated, but now by cooling down the reservoir. Here, the Seebeck coefficient follows the behavior of a resonant model with two extrema at $\varepsilon_{d}= \pm \gamma / 2$. All these differences for $S\left(\varepsilon_{d}\right)$ depending on the value of $\varepsilon_{M}$ could allow us to distinguish situations in which nanowires host true Majorana edge states or not.

\section{CONCLUSION}

We have investigated the linear response conductances to thermal and electrical biases in two-terminal geometries 
with topological superconductor nanowires hosting Majorana states. Firstly, we have considered a nanowire directly coupled to two normal reservoirs. Due to the intrinsic particle-hole symmetry this system exhibits a null thermopower: no voltage is generated in response to a thermal gradient. Then, we insert a quantum dot between the two normal contacts and the Majorana nanowire is side-coupled to the dot. With this arrangement the detection of the Majorana edge states can be performed by looking at the sign change of the thermoelectrical conductance or the thermopower $S$ with respect to $\varepsilon_{d}$ and $\varepsilon_{M}$. Besides, we show that both the electric and thermal conductances take their half-fermionic values whenever a true Majorana fermion state is formed.

We believe that our results could serve as a tool for the detection of Majorana edge states in semiconductor nanowires. Interestingly, possible changes of this physical scenario due to the Coulomb blockade effects or Kondo correlations are very interesting prospects for analysis in the future.
During the completion of this paper we become aware of a related work dealing with thermolectric transport in normal-dot-Majorana nanowires systems. The difference is that we consider thermal and electrical bias applied to the normal contacts; in Ref. [58] the thermoelectrical forces are applied to the normal and Majorana parts.

\section{ACKNOWLEDGMENTS}

We thank David Sánchez for useful discussions. This work was supported by MINECO Grant No. FIS201123526, in part by the Kavli Institute for Theoretical Physics through NSF Grant No. PHY11-25915, and by the National Research Foundation of Korea (NRF) grants funded by the Korea government (MSIP) (No. 2011-0030046).
[1] A. Dhar, Adv. Phys. 57, 457 (2008).

[2] Y. Dubi and M. Di Ventra, Rev. Mod. Phys. 83, 131 (2011).

[3] L. W. Molenkamp, Th. Gravier, H. van Houten, O. J. A. Buijk, M. A. A. Mabesoone, and C. T. Foxon, Phys. Rev. Lett. 68, 3765 (1992).

[4] A. S. Dzurak, C. G. Smith, C. H. W. Barnes, M. Pepper, L. Martín-Moreno, C. T. Liang, D. A. Ritchie, and G. A. C. Jones, Phys. Rev. B 55, R10197 (1997).

[5] S. F. Godijn, S. Möller, H. Buhmann, L. W. Molenkamp, and S. A. van Langen, Phys. Rev. Lett. 82, 2927 (1999).

[6] J. Matthews, D. Sánchez, M. Larsson, and H. Linke, Phys. Rev. B 85, 205309 (2012).

[7] P. N. Butcher, J. Phys.: Condens. Matter 2, 4869 (1990).

[8] P. Coleman, J. B. Marston, and A. J. Schofield, Phys. Rev. B 72, 245111 (2005).

[9] Ph. Jacquod and R. Whitney, Europhys. Lett. 91, 67009 (2010).

[10] V. Balachandran, R. Bosisio, and G. Benenti, Phys. Rev. B 86, 035433 (2012).

[11] P. Reddy, S.-Y. Jang. R. A. Segalman, and A. Majumdar, Science 315, 1568 (2007).

[12] F. Wilczek, Nat. Phys. 5, 614 (2009).

[13] G. Moore and N. Read, Nucl. Phys. B 360, 362 (1991).

[14] A. Y. Kitaev, Phys. Usp. 44, 131 (2001).

[15] D. A. Ivanov, Phys. Rev. Lett. 86, 268 (2001).

[16] S. Das Sarma, C. Nayak, and S. Tewari, Phys. Rev. B 73, 220502 (2006).

[17] J. Linder, Y. Tanaka, T. Yokoyama, A. Sudbø, and N. Nagaosa, Phys. Rev. Lett. 104, 067001 (2010).

[18] L. Fu and C. L. Kane, Phys. Rev. Lett. 100, 096407 (2008).

[19] Y. Oreg, G. Refael, and F. von Oppen, Phys. Rev. Lett. 105, 177002 (2010).

[20] J. Alicea, Phys. Rev. B 81, 125318 (2010).

[21] R. M. Lutchyn, J. D. Sau, and S. Das Sarma, Phys. Rev. Lett. 105, 077001 (2010).

[22] J. Linder and A. Sudbø, Phys. Rev. B 82, 085314 (2010).

[23] A. C. Potter and P. A. Lee, Phys. Rev. B 83, 184520 (2011).

[24] V. Mourik, K. Zuo, S. M. Frolov, S. R. Plissard, E. P. A. M. Bakkers, and L. P. Kouwenhoven, Science 336, 1003 (2012).
[25] M. T. Deng, C. L. Yu, G. Y. Huang, M. Larsson, P. Cardoff, and H. Q. Xu, Nano Lett. 12, 6414 (2012).

[26] A. Das, Y. Ronen, Y. Most, Y. Oreg, M. Heinblum, and H. Shtrikman, Nat. Phys. 8, 887 (2012).

[27] H. O. H. Churchill, V. Fatemi, K. Grove-Rasmussen, M. T. Deng, P. Caroff, H. Q. Xu, and C. M. Marcus, Phys. Rev. B 87, 241401 (2013).

[28] A. D. K. Finck, D. J. van Harlingen, P. K. Mohseni, K. Jung, and X. Li, Phys. Rev. Lett. 110, 126406 (2013).

[29] J. Liu, A. C. Potter, K. T. Law, and P. A. Lee, Phys. Rev. Lett. 109, 267002 (2012).

[30] F. Pientka, G. Kells, A. Romito, P. W. Brouwer, and F. von Oppen, Phys. Rev. Lett. 109, 227006 (2012).

[31] E. Prada, P. San-Jose, R. Aguado, Phys. Rev. B 86, 180503(R) (2012).

[32] J. S. Lim, R. López, and L. Serra, New J. Phys. 14, 083020 (2012).

[33] W. Chang, V. E. Manucharyan, T. S. Jespersen, J. Nygård, and C. M. Marcus, Phys. Rev. Lett. 110, 217005 (2013).

[34] E. J. H. Lee, X. Jiang, R. Aguado, G. Katsaros, C. M. Lieber, and S. De Franceschi, Phys. Rev. Lett. 109, 186802 (2012).

[35] G. Kells, D. Meidan, and P. W. Brouwer, Phys. Rev. B 86, 100503 (2012).

[36] E. J. H. Lee, X. Jiang, M. Houzet, R. Aguado, C. M. Lieber, and S. De Franceschi, Nature Nanotechnology 9, 79 (2014).

[37] D. I. Pikulin, J. P. Dahlhaus, M. Wimmer, H. Schomerus, and C. W. J. Beenakker, New. J. Phys. 14, 125011 (2012).

[38] H. J. Kwon, K. Sengupta, and V. M. Yakovenko, Eur. Phys. J. B 37, 349 (2003).

[39] L. Fu and C. L. Kane, Phys. Rev. B 79, 161408(R) (2009).

[40] Y. Tanaka, T. Yokoyama, and N. Nagaosa, Phys. Rev. Lett. 103, 107002 (2009).

[41] P. A. Ioselevich and M. V. Feigelman, Phys. Rev. Lett. 106, 077003 (2011).

[42] L. Jiang, D. Pekker, J. Alicea, G. Refael, Y. Oreg, and F. von Oppen, Phys. Rev. Lett. 107, 236401 (2011).

[43] D. I. Pikulin and Y. V. Nazarov, JETP Lett. 94, 693 (2012). 
[44] P. San-Jose, E. Prada, and R. Aguado, Phys. Rev. Lett. 108, 257001 (2012).

[45] F. Domínguez, F. Hassler, and G. Platero, Phys. Rev. B 86, 140503(R) (2012).

[46] M. Lee, H. Khim, and M.-S. Choi, Phys. Rev. B 89, 035309 (2014).

[47] L. Rokhinson, X. Liu, and J. K. Furdyna, Nat. Phys. 8, 795 (2012).

[48] K. Yang and B. I. Halperin, Phys. Rev. B 79, 115317 (2009).

[49] Y. Barlas and K. Yang, Phys. Rev. B 85, 195107 (2012).

[50] W. E. Chickering, J. P. Eisenstein, L. N. Pfeiffer, and K. W. West, Phys. Rev. B 87, 075302 (2013).
[51] C.-Y. Hou, K. Shtengel, and G. Refael, Phys. Rev. B 88, 075304 (2013).

[52] L. Onsager, Phys. Rev. 38, 2265 (1931).

[53] H. B. G. Casimir, Rev. Mod. Phys. 17, 343 (1945).

[54] M. Leijnse and K. Flensberg, Phys. Rev. B 84, 140501 (2011).

[55] D. E. Liu and H. U. Baranger, Phys. Rev. B 84, 201308 (2011).

[56] M. Lee, J. S. Lim, and R. López, Phys. Rev. B 87, 241402 (2013).

[57] A. R. Akhmerov, J. P. Dahlhaus, F. Hassler, M. Wimmer, and C. W. J. Beenakker, Phys. Rev. Lett. 106, 057001 (2011).

[58] M. Leijnse, New J. Phys. 16, 015029 (2014). 